\title{
The Use of the Esclera Scleral Contact Lens in the Treatment of Moderate to Severe Dry Eye Disease
}

\section{SARAH LA PORTA WEBER, RODRIGO BECCO DE SOUZA, JOSÉ ÁLVARO PEREIRA GOMES, AND ANA LUISA HOFLING-LIMA}

- PURPOSE: To evaluate the efficacy of the Esclera scleral contact lens (SCL) treatment and its impact on clinical testing for moderate to severe dry eye disease (DED).

- DESIGN: Prospective interventional case series.

- METHODS: A total of 41 eyes from 25 patients with moderate to severe DED were evaluated for the Esclera SCL treatment. Best-corrected visual acuity (BCVA), tear osmolarity, the Schirmer I test, tear film breakup time (TBUT), corneal and conjunctival staining, meibomian grading, and Ocular Surface Disease Index and SF-36v2 questionnaires were assessed before and after the SCL treatment. These values were compared to assess the real benefit of using SCL as a treatment for DED.

- RESULTS: Forty-one eyes from 25 patients were fitted with SCL for management of DED. The underlying diseases were Stevens-Johnson syndrome ( 22 eyes), Sjogren syndrome (11 eyes), graft-vs-host disease ( 2 eyes), dry eye after keratomileusis in situ ( 2 eyes), and undifferentiated ocular surface disease (4 eyes). BCVA improved from $0.703 \pm 0.55 \operatorname{logMAR}$ with habitual correction to $0.406 \pm 0.43 \log$ MAR with SCL $(P<.001)$. There was a significant decrease in tear osmolarity values $(338.1 \pm 27.1$ to $314.25 \pm 38.8 \mathrm{mOsm} / \mathrm{L}, P<.001)$ and van Bijsterveld scores $(3.63 \pm 2.33$ to $2.63 \pm 2.46$ grade, $P=.015)$ between the baseline and 12 months after SCL wear. There were also significant improvements in dry eye symptoms and quality of life as assessed by the OSDI and SF-36v2 questionnaires (both with $P<.001)$.

- CONCLUSIONS: The Esclera SCL treatment had a positive impact on tear osmolarity and van Bijsterveld score, as well as an improvement in the patients' BCVA, dry eye symptoms, and quality of life. (Am J Ophthalmol 2016;163:167-173. (C) 2016 by Elsevier Inc. All rights reserved.)

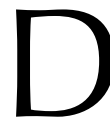

RY EYE DISEASE (DED) IS DEFINED AS "A MULTIFACtorial disease of the tears and ocular surface that results in symptoms of discomfort, visual

Accepted for publication Nov 25, 2015.

From the Department of Ophthalmology, Ocular Surface Advanced Center, Federal University of Sao Paulo, Sao Paulo, Brazil.

Inquiries to Sarah La Porta Weber, Avenida das Ubaranas, 1385, bloco 14, apart 303, Porto das Dunas, Aquiraz-CE, 61700-000, Brazil; e-mail: sarahlpweber@gmail.com disturbance, and tear film instability, with potential damage to the ocular surface." DED is accompanied by increased osmolarity of the tear film and inflammation of the ocular surface and is a common disorder that occurs more frequently in women than in men. This disorder affects a significant percentage of the population, especially among adults over 40 years of age. The prevalence is similar across countries worldwide, with rates between $7 \%$ and $33 \% .^{2}$

Conventional treatments for DED include the application of artificial tears, topical corticosteroids or cyclosporin A, therapeutic soft contact lenses, and surgical procedures such as punctal occlusion and tarsorrhaphy. ${ }^{3,4}$ However, despite these therapies, many patients complain of persistent symptoms and continue to show signs associated with ocular surface changes. Additionally, the use of scleral contact lenses (SCLs) has been recognized as an alternative for the treatment of these patients. ${ }^{5-10}$

SCLs are large-diameter, rigid, gas-permeable devices that are completely supported by the sclera and that vault the cornea and limbus. SCLs maintain a fluid reservoir in the space between the posterior surface of the lens and the anterior surface of the cornea. The unique fitting characteristics of SCLs enable the protection of the ocular surface from shear forces generated by eyelid movement over the cornea and provide continuous hydration of the ocular surface. $^{10,11}$

The purpose of this study was to evaluate the impact of wearing the Esclera SCL on dry eye and quality of life in patients with moderate to severe DED.

\section{METHODS}

THIS PROSPECTIVE INTERVENTIONAL CASE SERIES WAS approved by the Ethics Committee of Federal University of Sao Paulo (Sao Paulo, Brazil). Informed consent was obtained from all participants after the nature and possible consequences of the study were explained, and the research was carried out according to the tenets of the Declaration of Helsinki.

This study evaluated 41 eyes from 25 patients who were fitted with Esclera SCLs to treat moderate to severe DED. When a patient had an indication for SCL in both eyes, 
we analyzed the values for each eye as independent variables, considering that each eye presents independent anatomic and physiological features. These patients were referred to the Contact Lens Department of Federal University of Sao Paulo (Sao Paulo, Brazil) from February 4, 2013 to November 28, 2014. The mean age was 39.51 \pm 12.16 years, and $26(63.4 \%)$ eyes of patients were female. No patients were lost to follow-up.

This study included patients with grades 2, 3, and 4 DED based on the DEWS report, also known as moderate to severe DED, which presents occasionally annoying or constant visual symptoms, changes in conjunctival staining and injection, changes in corneal staining and tear signs, changes in the meibomian glands, a tear film breakup time (TBUT) $\leq 10$ seconds, and a Schirmer score $\leq 10.1$ The patients evaluated had symptoms that could not be controlled by conventional treatments.

Patients with the following conditions were excluded from the study: glaucoma, disorders that affect sensitivity (eg, herpetic disease and diabetes mellitus), corneal decompensation, active ocular infection, anatomic variations of the eyelid and conjunctiva that impair proper SCL fitting, pregnancy, and an inability to correctly handle and care for the SCLs.

For SCL fitting, a trial set with the following parameters was used: scleral design (Esclera; Mediphacos Inc, Belo Horizonte, Brazil); nonfenestrated; diameter, 16-18.2 mm; available sagittal vaults, 4.12-6.27 mm; $\mathrm{DK} / \mathrm{T}, 141$ (ISO/Fatt); available powers from -20 to +20 diopters.

All SCL fittings were performed by a practitioner experienced in the field (S.L.P.W.). The 3 parameters that characterize the Esclera SCL are the sagittal depth, base curve, and lens diameter. The initial diagnostic lens was selected based on suggestions in the manufacturer's fitting guide ${ }^{12}$ according to the patient's corneal topographic diagnosis, such as moderate cone, advanced cone, pellucid marginal degeneration, or post-penetrating keratoplasty.

The ideal Esclera SCL for fitting had a size at least $2 \mathrm{~mm}$ greater than each side of the limbus and a minimum apical clearance of $100 \mu \mathrm{m}$. The SCL should not touch the cornea, and the edges of the SCL should not exhibit vascular impingement, conjunctival blanching, or scleral indentation. Those patients with ideal fits were allowed to wear the lenses for 1 hour; then, the apical clearance was reassessed, and spherocylindrical over-refraction was performed.

Follow-up visits occurred at months $0,1,3,6$, and 12 . Subjective and objective assessments of DED were conducted before the fitting and 6 and 12 months after SCL use. All dry eye tests were performed by the same observer (S.L.P.W.), and the minimum follow-up period was 12 months.

The clinical examinations included assessment of the best-corrected visual acuity (BCVA) using Early Treatment Diabetic Retinopathy Study (ETDRS) charts
(CC-100; Topcon Corp, Tokyo, Japan), and this value was recorded as the Snellen equivalent. The corresponding $\operatorname{logMAR}$ was then derived from the Snellen equivalent.

At each visit, in order of performance, tear osmolarity, slit-lamp examination, the Schirmer I test, the TBUT, corneal and conjunctival staining, and meibomian grading were assessed for the eyes evaluated. All patients were instructed to discontinue use of the SCLs 1 day prior to dry eye testing.

Osmolarity was measured using a lab-on-a-chip system to simultaneously collect and analyze the electrical impedance of a $50-\mathrm{nL}$ tear sample from the inferior lateral meniscus (TearLab Osmolarity System; OcuSense, San Diego, California, USA). ${ }^{13,14}$ We excluded patients with Schirmer I test scores equal to zero from this analysis.

A slit-lamp examination at a magnification of $10-16 \times$ was used to detect the presence of active inflammation or structural changes, as evidenced by scarring in the eyelid and conjunctiva, neovascularization, opacities, or thinning of the cornea. The tear meniscus height was classified as present, reduced, or absent.

Tear fluid production was examined with a 5 -minute Schirmer I test using a standardized filter strip (Ophthalmos Inc, Sao Paulo, Brazil) without anesthetic. The TBUT was measured by calculating the average of 3 consecutive tear breakup times, which were determined manually using a stopwatch.

Corneal staining was evaluated after fluorescein instillation according to the van Bijsterveld score (VBS) ${ }^{15}$ (grades of $0-3$ for 3 regions of the ocular surface). Conjunctival and corneal staining with sodium lissamine green dye was assessed using the Oxford score, ${ }^{16}$ for which grades of $0-5$ are assigned to 3 regions of the ocular surface for a possible total of 15 points.

For meibomian gland evaluation, digital pressure was applied to the upper tarsus, and meibum expression was evaluated semiquantitatively according to the following grades: 0 , clear meibum easily expressed; 1 , cloudy meibum expressed with mild pressure; 2 , cloudy meibum expressed with more than moderate pressure; 3, meibum not expressed even with strong pressure. ${ }^{17}$

Symptoms were assessed at baseline and after 12 months of SCL use using a validated Portuguese version of the Ocular Surface Disease Index (OSDI) (Allergan Inc, Irvine, California, USA). The OSDI scores range from 0 to 100 , with higher scores representing greater disability. ${ }^{18}$ In addition, the SF-36v2 questionnaire (QualityMetric Inc, Lincoln, Rhode Island, USA) ${ }^{19}$ was used to assess patient quality of life (QoL) before and after SCL use. For each domain, a score ranging from 0 (worst health) to 100 (best health) was calculated.

- STATISTICAL ANALYSIS: Statistical analyses were performed with the statistical software package SPSS for Windows (version 14.0; SPSS, Inc, Chicago, Illinois, USA). To compare the results obtained at baseline and 
at 6 and 12 months after SCL use, we performed the Cochran test, the Friedman test, analysis of variance, and paired $t$ tests, depending on the variable analyzed. Normality assumption was evaluated by the Shapiro-Wilk test. In addition, when necessary, Tukey multiple comparisons were performed. Differences were considered statistically significant when the $P$ value was less than .05 .

\section{RESULTS}

THIS STUDY EVALUATED 41 EYES FROM 25 PATIENTS WHO were fitted with SCLs to treat DED. Demographic information on all subjects is summarized in Table 1 . The topical dry eye therapy and ocular surgeries attempted before the SCL evaluation are described in Table 2 . We noted that most of the patients had previously undergone certain treatment for DED, as suggested by DEWS. ${ }^{1}$ Another group of potential subjects (15-20 eyes) attempted to enroll in the study but could not participate because they were unable to handle the contact lens.

TABLE 1. Demographic Data of Dry Eye Disease Patients Evaluated for Esclera Scleral Contact Lens Therapy

\begin{tabular}{|c|c|}
\hline Demographic & Value \\
\hline Eyes/patients (n) & $41 / 25$ \\
\hline \multicolumn{2}{|l|}{ Laterality of fit (n) } \\
\hline Right & 4 \\
\hline Left & 5 \\
\hline Both & 16 \\
\hline \multicolumn{2}{|l|}{ Age at SCL fitting (y) } \\
\hline Mean \pm SD & $39.51 \pm 12.158$ \\
\hline Range & 44 \\
\hline Female/male sex (\%) & $\begin{array}{c}26 \text { eyes }(63.4) / \\
15 \text { eyes }(36.6)\end{array}$ \\
\hline \multicolumn{2}{|l|}{ UDVA (logMAR) } \\
\hline Mean \pm SD & $1.047 \pm 0.538$ \\
\hline Range & 1.90 \\
\hline \multicolumn{2}{|c|}{ BCVA with habitual correction (logMAR) } \\
\hline Mean \pm SD & $0.703 \pm 0.555$ \\
\hline Range & 2.00 \\
\hline \multicolumn{2}{|c|}{ Previous spectacle wear (n/\%) } \\
\hline Yes & $16 / 39$ \\
\hline No & $25 / 61$ \\
\hline \multicolumn{2}{|c|}{ Previous contact lens wear (n/\%) } \\
\hline Yes & $21 / 51.2$ \\
\hline No & $20 / 48.8$ \\
\hline \multicolumn{2}{|l|}{ Dry eye grading $^{a}(\%)$} \\
\hline Grade 1 & 0 \\
\hline Grade 2 & 37.5 \\
\hline Grade 3 & 21.9 \\
\hline Grade 4 & 40.6 \\
\hline \multicolumn{2}{|c|}{$\begin{array}{l}\mathrm{BCVA}=\text { best-corrected visual acuity; } \mathrm{SCL}=\text { scleral contact } \\
\text { lens; UCVA = uncorrected distance visual acuity. } \\
{ }^{a} \text { According to DEWS. }{ }^{1}\end{array}$} \\
\hline
\end{tabular}

TABLE 2. Topical Dry Eye Disease Therapy and Ocular Surgeries Attempted Before the Esclera Scleral Contact Lens Evaluation

$\begin{array}{lc}\text { Prior topical therapy, } \mathrm{n} / \% \text { ( } \mathrm{n}=41 \text { eyes) } & \\ \text { Artificial tears preservative-free } & 39 / 95.1 \\ \text { Artificial tears with preservative } & 7 / 17.1 \\ \text { Gels/ointments } & 11 / 26.8 \\ \text { Topical antibiotic eye drops } & 10 / 24.4 \\ \text { Corticosteroid eye drops } & 24 / 58.5 \\ \text { Cyclosporine A 0.05\% eye drops } & 4 / 9.75 \\ \text { Autologous serum tears } & 1 / 2.4 \\ \text { Prior ocular surgery, } \mathrm{n} / \% & \\ \text { Punctal occlusion } & 14 / 34.1 \\ \text { Tarsorrhaphy } & 7 / 17.1 \\ \text { Penetrating keratoplasty } & 3 / 7.3 \\ \text { Eyelid reconstruction } & 10 / 24.4 \\ \text { Electrolysis of cilia } & 11 / 26.8 \\ \text { Salivary gland autotransplantation } & 7 / 17.1 \\ \text { Coating with amniotic membrane } & 2 / 4.9\end{array}$

The underlying diseases were Stevens-Johnson syndrome (22 eyes), Sjogren syndrome (11 eyes), graftvs-host disease (GVHD) (2 eyes), dry eye after in situ keratomileusis (LASIK) (2 eyes), and undifferentiated ocular surface disease (4 eyes).

The BCVA improved from $0.703 \pm 0.55 \log M A R$ (mean \pm SD; Snellen equivalent, 20/100) with habitual correction to $0.406 \pm 0.43 \operatorname{logMAR}$ (Snellen equivalent, 20/50) with the SCL $(P<.001)$. All patients who were fitted with SCLs had an improved BCVA, defined as a gain of 2 or more Snellen lines. The mean SCL wear time per day was $11.6 \pm 3.0$ hours (range, 5-15 hours).

The slit-lamp findings present at baseline were corneal neovascularization $(77.8 \%)$, corneal opacity $(61.1 \%)$, corneal thinning $(30.6 \%)$, and corneal keratinization $(16.7 \%)$. Eyelid scarring was present in $41.7 \%$ of the eyes studied. None of the slit-lamp findings showed changes between the baseline and the 12-month evaluations $(P=1.000)$. The status of the meibomian glands was $2.8 \%$ grade $0+, 50 \%$ grade $1+, 44.4 \%$ grade $2+$, and $2.8 \%$ grade $3+$ (Table 3 ). The analysis of the tear meniscus height showed that 15 eyes $(36.6 \%)$ had no meniscus, $22(53.6 \%)$ had a reduced meniscus, and $4(9.8 \%)$ had a present meniscus at baseline. These results showed no changes between the baseline data and those data obtained after 6 and 12 months $(P=1.000)$.

The parameters of the final Esclera SCL fitting were a mean lens sagittal depth of $4.74 \pm 0.38 \mathrm{~mm}$ and a mean lens base curve of $7.28 \pm 0.57 \mathrm{~mm}$. The diameter of the fitted SCL ranged from 16.0 to $17.5 \mathrm{~mm}$, with an average of $16.43 \mathrm{~mm}$.

Table 3 shows the outcomes of the DED testing at baseline and then 6 months and 12 months after SCL wear. We noticed that tear osmolarity and the VBS were significantly different between the analyzed periods $(P<.001$ 
TABLE 3. Dry Eye Disease Testing Outcomes at Baseline and 6 and 12 Months After Esclera Scleral Contact Lens Wear

\begin{tabular}{lcccc}
\hline & Baseline $(\mathrm{n}=41)$ & 6 Months $(\mathrm{n}=41)$ & 12 Months $(\mathrm{n}=41)$ & $P$ Value \\
\hline Tear osmolarity (mOsm/L) & $338.1 \pm 27.1$ & $313.1 \pm 44.1$ & $314.25 \pm 38.8$ & $<.001^{a}$ \\
Schirmer I test (mm/5 min) & $3.2 \pm 3.443$ & $2.85 \pm 3.407$ & $2.5 \pm 3.204$ & .372 \\
TBUT value (s) & $2.65 \pm 1.785$ & $2.9 \pm 1.586$ & $2.9 \pm 1.518$ & .555 \\
van Bjisterveld score (grade) & $3.63 \pm 2.337$ & $3.04 \pm 2.458$ & $2.63 \pm 2.464$ & $.015^{a}$ \\
Oxford score (grade) & $5.33 \pm 3.975$ & $4.5 \pm 2.485$ & $4.42 \pm 2.376$ & .209 \\
Meibomian gland status (grade) & $2.00 \pm 0$ & $2.00 \pm 0$ & $2.00 \pm 0$ & 1.000 \\
\hline
\end{tabular}

TBUT $=$ tear film breakup time.

Abnormal values: Tear osmolarity $\geq 316 \mathrm{mOsm} / \mathrm{L}$; Schirmer I test $\leq 5 \mathrm{~mm} / 5 \mathrm{~min}$; TBUT $<10$ seconds.

Values expressed as mean \pm standard deviation.

${ }^{a}$ Statistically significant correlation $(P<.05)$; analysis by repeated-measures analysis of variance test.

and $P=.015$, respectively). Eight eyes were excluded from the tear osmolarity analysis because they presented Schirmer I test scores equal to zero for at least 1 of the measurements.

The tear osmolarity exhibited a statistically significant decrease between different time points: baseline $>$ 6 months $(P<.001)$, baseline $>12$ months $(P<.001)$, and 6 months $=12$ months $(P=.929)$, as determined using the Tukey test at a $1 \%$ level of probability. The statistical significance of the differences in the VBS between different time points were as follows: baseline $=6$ months $(P=$ $.194)$, baseline $>12$ months $(P=.011)$, and 6 months $=$ 12 months $(P=.426)$, as determined using the Tukey test at a $1 \%$ level of probability.

The other DED tests shown in Table 3, such as the Schirmer I test, TBUT assessment, the Oxford score, and assessment of the meibomian gland status, showed no significant difference between baseline and after 6 and 12 months of SCL wear.

Ocular surface symptoms assessed by OSDI score were significantly better after 12 months of SCL wear $(P<.001)$ (Table 4). The patient QoL assessed by SF-36v2 questionnaire was also significantly better after 12 months of SCL wear in the 8 domains evaluated $(P<.001)$ as described in Table 4.

The mean follow-up period was 16.3 months (range, 12.2-24.5 months). During the follow-up, 1 eye (2.44\%) presented with a corneal abrasion during lens insertion, and no patients discontinued SCL wear.

\section{DISCUSSION}

THE 2 MAIN MECHANISMS OF DED ARE DRIVEN BY TEAR hyperosmolarity and tear film instability. Tear hyperosmolarity arises as a result of water evaporation from the exposed ocular surface, low aqueous tear flow, or excessive evaporation or owing to a combination of these events. Tear film instability also suggests higher exposure of the ocular surface, resulting in damage to the epithelial surface and the glycocalyx and goblet cell mucin disorders. ${ }^{1}$ This process is accompanied by ocular surface inflammation, which causes dysfunction of the lacrimal glands, changing the composition to a state of tear hyperosmolarity, and then completes the vicious circle of tissue damage. ${ }^{20}$ The degree of tear film hyperosmolarity has been proven to be the most effective single measure for diagnosing DED, ${ }^{21-23}$ and moderate to severe dry eye is characterized by tear osmolarity higher than $316 \mathrm{mOsm} / \mathrm{L} .^{13,23,24}$

Over the past decade, several studies have investigated different SCLs for the treatment of DED. ${ }^{5-8,10,25-33}$ This interest is due to the introduction of new SCL designs and the improvement of SCL materials and oxygen permeability. The use of SCLs is usually indicated for treating $\mathrm{DED}$ in cases of conventional treatment

TABLE 4. Questionnaire Evaluation of Dry Eye Disease Patients Before and 12 Months After Esclera Scleral Contact Lens Wear

\begin{tabular}{|c|c|c|c|}
\hline Questionnaire & Baseline $(n=25)$ & $\begin{array}{l}\text { After } 12 \text { Months } \\
\qquad(\mathrm{n}=25)\end{array}$ & $P$ Value $^{a}$ \\
\hline OSDI & $30.71 \pm 14.13$ & $11.29 \pm 11.24$ & $<.001$ \\
\hline \multicolumn{4}{|l|}{ SF-36v $2^{b}$} \\
\hline Physical functioning & $91.25 \pm 3.24$ & $97.47 \pm 5.22$ & $<.001$ \\
\hline Social functioning & $73.38 \pm 21.32$ & $84.55 \pm 18.96$ & $<.001$ \\
\hline Physical problems & $54.37 \pm 34.14$ & $61.46 \pm 40.33$ & $<.001$ \\
\hline Bodily pain & $66.48 \pm 21.94$ & $72.28 \pm 23.46$ & $<.001$ \\
\hline Emotional problems & $55.55 \pm 41.14$ & $68.75 \pm 43.87$ & $<.001$ \\
\hline Mental health & $65.55 \pm 12.90$ & $74.12 \pm 19.02$ & $<.001$ \\
\hline Energy and vitality & $59.08 \pm 10.80$ & $68.76 \pm 12.40$ & $<.001$ \\
\hline $\begin{array}{l}\text { General perception } \\
\text { of health }\end{array}$ & $48.55 \pm 15.89$ & $62.09 \pm 18.33$ & $<.001$ \\
\hline
\end{tabular}

OSDI = Ocular Surface Disease Index.

Values expressed as mean \pm standard deviation.

aPaired $t$ test.

${ }^{b} 1998$ US general population norms and norm-based scoring. ${ }^{17}$ 
failure. ${ }^{10,29}$ The SCL acts as a protective covering for the cornea and conjunctivae by controlling evaporation and maintaining direct contact between the fluid and the corneal epithelium. The SCL also protects the cornea from abrasions and mechanical trauma, which commonly result from eyelid scar irregularities and misdirected eyelashes.

The present study evaluated the impact of the Esclera SCL on dry eye and quality of life in patients with moderate to severe DED. Of the 41 eyes assessed in this study, $62.5 \%$ were classified as grade 3 or 4 according to DEWS. ${ }^{1}$ This percentage explains the severity of the slit-lamp findings and the DED testing outcomes (Table 3 ) observed during the study. Another group of potential subjects (15-20 eyes) attempted to enroll in the study but could not participate because they were unable to handle the contact lens. The main difficulty was lens placement in the eye by the patients. Given that the SCL is a large-diameter lens and that it should be completely filled with liquid, its placement requires manual skill. Other factors, such as low vision, a lack of previous experience with contact lenses, hand tremors, anatomic abnormalities of the fingers (eg, osteoarthritis), or insecurity due to living alone were decisive for the exclusion of these patients from our study.

Most of the patients evaluated had Stevens-Johnson syndrome $(53.7 \%)$ or Sjogren syndrome (26.8\%). Our study institution is one of the national reference centers for treating these diseases.

We noticed a significant improvement in the BCVA (gain of 2 or more Snellen lines) in all patients who were fitted with SCLs, as also described in previous studies. ${ }^{5,10,11,29-33}$ In addition to correcting the refraction of the patient, the SCL promotes the replacement of an irregular ocular surface, with findings such as opacities, scarring, and corneal neovascularization, with a more regular surface. The improvement in the quality of the BCVA could also have been the result of reducing the dry eye symptoms, including irritation, photophobia, and foreign-body sensation, described in the OSDI questionnaire (Table 4), which increases comfort when the eyes are open.

In our study, the diameter of the fitted SCL ranged from 16.0 to $17.5 \mathrm{~mm}$, with an average of $16.43 \mathrm{~mm}$. Previous studies have investigated even larger lenses, with diameters ranging from 15.0 to $23.0 \mathrm{~mm} .^{5,9-11,26-32}$ We believe that the diameter of the lens used in the present study facilitated successful SCL fittings in patients with eyelid scarring $(41.7 \%)$.

We observed a statistically significant decrease in the mean tear osmolarity between baseline and after 6 months, and this decrease was maintained after 12 months of SCL wear. The reduction in tissue damage, as assessed based on van Bijsterveld corneal staining, showed a downward trend; this trend was not significant after six months of SCL wear but became significant after 12 months. This finding may suggest that the process of tecidual damage was still active during and after normalization of the tear osmolarity. The SCL reduces tear evaporation, which allows several of the tear properties to be gradually reestablished, regardless of the etiology of the dry eye.

However, several of the measurement parameters related to tear film instability, such as the Schirmer I test results and the TBUT, exhibited no statistically significant changes between periods evaluated, as shown in Table 3 . Therefore, the use of the SCL allowed only a partial increase in ocular surface epithelialization because it reverted just 1 of the 2 core mechanisms of damagenamely, the hyperosmolarity-and did not alter the tear film instability.

According to the OSDI, most patients experienced many symptoms associated with dry eye, such as foreignbody sensation, ocular fatigue, and eye redness. However, compared with the value before SCL use, the OSDI showed consistent improvement after SCL use. The average OSDI value ranged from 30.71 to 11.29 after 12 months of SCL use $(P<.001)$, with a higher value indicating more severe ocular complaints related to dry eye. Previous studies have shown a significant improvement between pre-SCL and post-SCL OSDI scores, which indicates that the patients experienced improvements in ocular surface comfort and visual function. ${ }^{21}$ These results may explain why despite the disadvantages of the lens, such as difficulties in handling and care, patients maintained their use during the follow-up period. In addition, we observed a mean duration of SCL wear per day of $11.6 \pm 3.0$ hours (range, 5-15 hours), which also demonstrates the adherence to DED treatment with the SCL.

The patient QoL assessed by SF-36v2 questionnaire was also significantly better after 12 months of SCL wear in each of the 8 domains evaluated $(P<.001)$. The SF-36 was chosen because it is one of the most widely used measures in health services research and has already been translated into the Portuguese language and validated. ${ }^{19,34,35}$ Dry eye disease is clearly associated with poorer QoL, with particular impact on the physical component summary. ${ }^{36}$ In our study, the symptoms of moderate to severe dry eye and its primary diseases affected the QoL in general, especially involving the physical problems, bodily pain, emotional problems, mental health, energy and vitality, and the general perception of health (Table 4). This demonstrates that moderate to severe DED has huge impact on the patients' quality of life.

Our study had certain limitations, such as the small sample of eyes evaluated and the lack of a control group. In addition, we analyzed the values for each eye as independent variables, which could lead to some bias. However, despite these limitations, we could demonstrate that Esclera SCL treatment had a positive impact on tear osmolarity and the VBS and also improved BCVA and patients' quality of life. Regardless, more studies with a larger sample size and longer-term follow-up are needed in this field to investigate the impact of SCLs. 
FUNDING/SUPPORT: SUPPORTED BY THE BRAZILIAN GOVERNMENT COORDENAÇÃO DE APERFEIÇOAMENTO DE PESSOAL DE Nível Superior-CAPES Foundation (Brasília, DF, Brazil [Sarah La Porta Weber]); and Mediphacos Inc. (Belo Horizonte, MG, Brazil). Financial disclosures: José Álvaro Pereira Gomes serves as a consultant for Allergan MSD (Irvine, California, USA); and on the lecture boards for Alcon (Fort Worth, Texas, USA), Allergan (Irvine, California, USA), Genon (São Paulo, SP, Brazil), Bausch \& Lomb (Rochester, New York, USA), and Pfizer (New York, New York, USA). The following authors have no financial disclosures: Sarah La Porta Weber, Rodrigo Becco de Souza, and Ana Luisa Hofling-Lima. All authors attest that they meet the current ICMJE criteria for authorship.

The authors gratefully thank and acknowledge Dr Rossen M. Hazarbassanov, PhD (Federal University of São Paulo, UNIFESP, São Paulo, Brazil) for support with osmolarity testing and Dr Taís H. Wakamatsu, PhD (Federal University of São Paulo, UNIFESP, São Paulo, Brazil) for support with dry eye testing.

\section{REFERENCES}

1. The definition and classification of dry eye disease: report of the Definition and Classification Subcommittee of the International Dry Eye WorkShop (2007). Ocul Surf 2007;5(2): 75-92.

2. Schein OD, Hochberg MC, Munoz B, et al. Dry eye and dry mouth in the elderly: a population-based assessment. Arch Intern Med 1999;159(12):1359-1363.

3. Lemp MA. Management of dry eye disease. Am J Manag Care 2008;14(3):S88-101.

4. Pflugfelder SC, Solomon A, Stern ME. The diagnosis and management of dry eye: a twenty-five-year review. Cornea 2000;19(5):644-649.

5. Romero-Rangel T, Stavrou P, Cotter J, Rosenthal P, Baltatzis S, Foster CS. Gas-permeable scleral contact lens therapy in ocular surface disease. Am J Ophthalmol 2000; 130(1):25-32.

6. Rosenthal P, Cotter J. The Boston Scleral Lens in the management of severe ocular surface disease. Ophthalmol Clin North Am 2003;16(1):89-93.

7. Rosenthal P, Croteau A. Fluid-ventilated, gas-permeable scleral contact lens is an effective option for managing severe ocular surface disease and many corneal disorders that would otherwise require penetrating keratoplasty. Eye Contact Lens 2005;31(3):130-134.

8. Rosenthal P, Cotter JM, Baum J. Treatment of persistent corneal epithelial defect with extended wear of a fluid ventilated gas-permeable scleral contact lens. Am J Ophthalmol 2000;130(1):33-41.

9. Jacobs DS, Rosenthal P. Boston scleral lens prosthetic device for treatment of severe dry eye in chronic graft-versus-host disease. Cornea 2007;26(10):1195-1199.

10. Schornack MM, Pyle J, Patel SV. Scleral Lenses in the Management of Ocular Surface Disease. Ophthalmology 2014;121(7):1398-1405.

11. van der Worp E, Bornman D, Ferreira DL, Faria-Ribeiro M, Garcia-Porta N, González-Meijome JM. Modern scleral contact lenses: a review. Cont Lens Anterior Eye 2014;37(4): 240-250.

12. FITTING GUIDE: Maxim ${ }^{\mathrm{TM}}$ Scleral Lens. Available at http://www.acculens.com/fitting_maxim.htm. Accessed July $12,2015$.

13. Tomlinson A, Khanal S, Ramaesh K, Diaper C, McFadyen A. Tear film osmolarity: determination of a referent for dry eye diagnosis. Invest Ophthalmol Vis Sci 2006;47(10): 4309-4315.

14. Farris RL, Stuchell RN, Mandel ID. Tear osmolarity variation in the dry eye. Trans Am Ophthalmol Soc 1986;84:250-268.
15. van Bijsterveld OP. Diagnostic test in the Sicca syndrome. Arch Ophthalmol 1969;82(1):10-14.

16. Bron AJ. The Doyne Lecture. Reflections on the tears. Eye 1997;11(Pt 5):583-602.

17. Shimazaki J, Sakata M, Tsubota K. Ocular surface changes and discomfort in patients with meibomian gland dysfunction. Arch Ophthalmol 1995;113(10):1266-1270.

18. Schiffman RM, Christianson MD, Jacobsen G, Hirsch JD, Reis BL. Reliability and validity of the Ocular Surface Disease Index. Arch Ophthalmol 2000;118(5):615-621.

19. Ware JE, Kosinski M, Bjorner JB, Turner-Bowker DM, Gandek B, Maruish ME. SF-36v2® Health Survey: administration guide for clinical trial investigators. Lincoln: QualityMetric Inc; 2008:1-44.

20. Moore QL, De Paiva CS, Pflugfelder SC. Effects of dry eye therapies on environmentally induced ocular surface disease. Am J Ophthalmol 2015;160(1):135-142.

21. Gilbard JP, Farris RL. Tear osmolarity and ocular surface disease in keratoconjunctivitis sicca. Arch Ophthalmol 1979; 97(9):1642-1646.

22. Bron AJ, Tiffany JM, Yokoi N, Gouveia SM. Using osmolarity to diagnose dry eye: a compartmental hypothesis and review of our assumptions. Adv Exp Med Biol 2002;506(Pt B):1087-1095.

23. Jacobi C, Jacobi A, Kruse FE, Cursiefen C. Tear film osmolarity measurements in dry eye disease using electrical impedance technology. Cornea 2011;30(12):1289-1292.

24. Sullivan BD, Whitmer D, Nichols KK, et al. An objective approach to dry eye disease severity. Invest Ophthalmol Vis Sci 2010;51(12):6125-6130.

25. Weyns M, Koppen C, Tassignon MJ. Scleral contact lenses as an alternative to tarsorrhaphy for the long-term management of combined exposure and neurotrophic keratopathy. Cornea 2013;32(3):359-361.

26. Takahide K, Parker PM, Wu M, et al. Use of fluid ventilated, gas-permeable scleral lens for management of severe keratoconjunctivitis sicca secondary to chronic graft-versushost disease. Biol Blood Marrow Transplant 2007;13(9): 1016-1021.

27. Pullum KW, Buckley RJ. A study of 530 patients referred for rigid gas permeable scleral contact lens assessment. Cornea 1997;16(6):612-622.

28. Pullum K, Buckley R. Therapeutic and ocular surface indications for scleral contact lenses. Ocul Surf 2007;5(1):40-48.

29. Jacobs DS. Update on scleral lenses. Curr Opin Ophthalmol 2008;19(4):298-301.

30. Kok JH, Visser R. Treatment of ocular surface disorders and dry eyes with high gas-permeable scleral lenses. Cornea 1992;11(6):518-522. 
31. Alipour F, Kheirkhah A, Jabarvand BM. Use of mini scleral contact lenses in moderate to severe dry eye. Cont Lens Anterior Eye 2012;35(6):272-276.

32. Papakostas TD, Le HG, Chodosh J, Jacobs DS. Prosthetic replacement of the ocular surface ecosystem as treatment for ocular surface disease in patients with a history of Stevens-Johnson syndrome/toxic epidermal necrolysis. Ophthalmology 2015;122(2):248-253.

33. Heur M, Bach D, Theophanous C, Chiu GB. Prosthetic replacement of the ocular surface ecosystem scleral lens therapy for patients with ocular symptoms of chronic Stevens-Johnson syndrome. Am J Ophthalmol 2014;158(1): 49-54.
34. McHorney CA, Ware JE Jr, Raczek AE. The MOS 36-Item Short-Form Health Survey (SF-36): II. Psychometric and clinical tests of validity in measuring physical and mental health constructs. Med Care 1993;31(3):247-263.

35. McHorney CA, Ware JE Jr, Lu JF, Sherbourne CD. The MOS 36-Item Short-Form Health Survey (SF-36): III. Tests of data quality, scaling assumptions, and reliability across diverse patient groups. Med Care 1994;32(1): 40-66.

36. Daikeler T, Mauramo M, Rovó A, et al. Sicca symptoms and their impact on quality of life among very long-term survivors after hematopoietic SCT. Bone Marrow Transplant 2013; 48(7):988-993. 


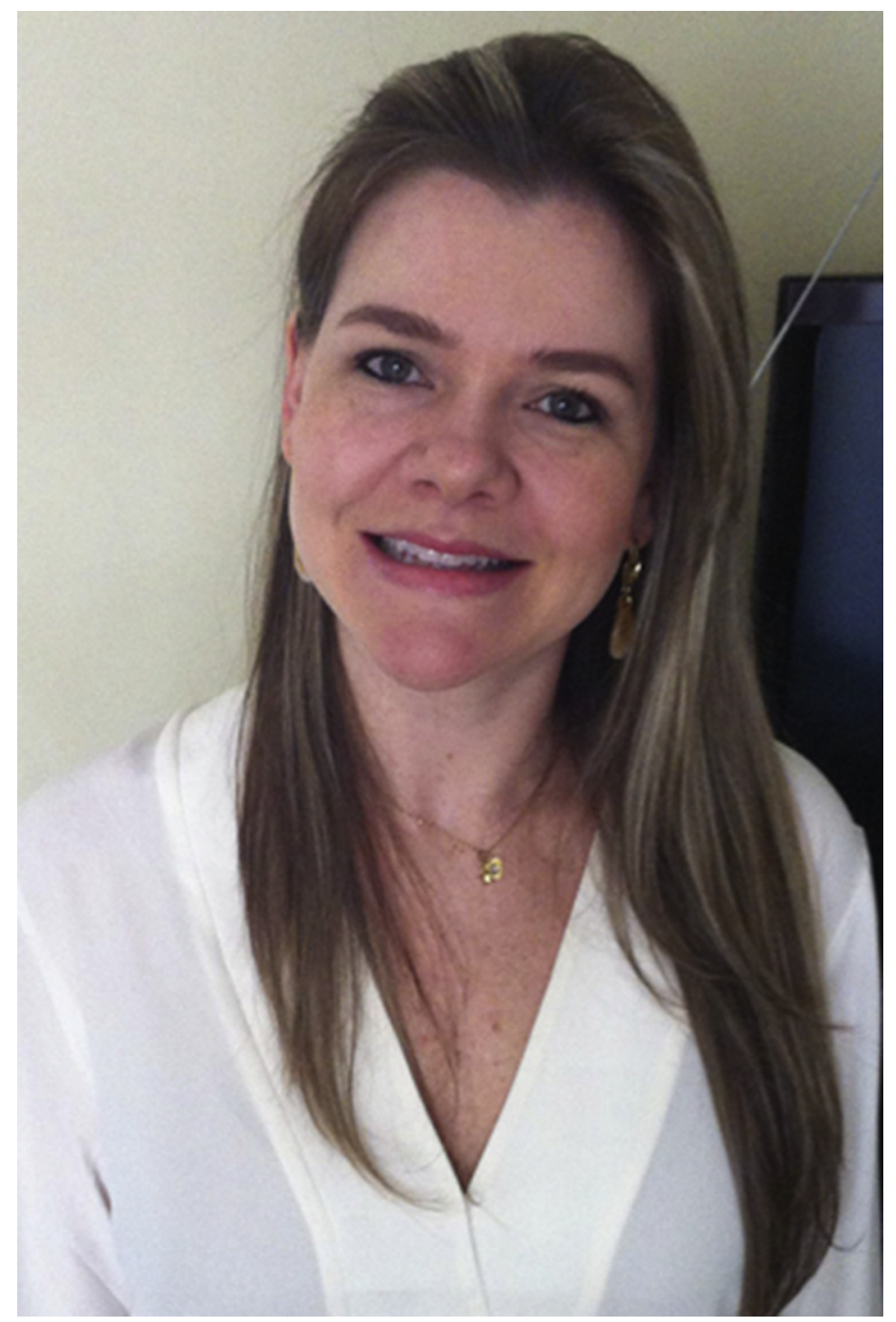

Biosketch

Sarah La Porta Weber, MD, received her medical degree from University of Joinville Region; and did his Ophthalmology residency at Santa Casa de São Paulo School of Medical Sciences. She completed a Cataract surgery Fellowship at University of Sao Paulo and Contact lens Fellowship at Federal University of Sao Paulo. Dr Weber is currently finishing her post-graduation at the Doctoral Programme of Federal University of Sao Paulo. Her reasearch interests are cataract surgery, contact lens and ocular surface. 